\title{
Purification of total flavonoids from loquat leaves by macroporous resin and corresponding antioxidant capacity
}

\author{
Suhua Huang, Biaosheng Lin, Binbin Li, Bi Tan and Yanping Hong ${ }^{a}$ \\ College of Life Science, Longyan University, Longyan, Fujian 364012, China
}

\begin{abstract}
Flavonoids is one of the major active compounds in loquat leaves. In this study, the purification process of total flavonoids in loquat leaves by macroporous resin was researched and the antioxidant activity of total flavonoids was determined. The active compounds were extracted by $95 \%$ ethanol, and the total flavonoids was purified by macroporous resin. Comparing the static and dynamic adsorption and desorption characters of 6 macroporous resin, the best type of macroporous resin was determined. The desorption-solvent was determined by the gradient elution. Moreover, DPPH and FRAP analysis methods were used to determine the antioxidant activity of total flavonoids in this study. The result showed that X-5 was the optimum macroporous resin to purify the total flavonoids of loquat leaf. After adsorbed by X-5 macroporous resin, removing impurity with $15 \%$ ethanol and desorbed with $65 \%$ ethanol, the total flavonoids purity of the eluate was $82.20 \%$, the purification factor was 2.14 times and the recovery was $81.08 \%$, and the macroporous resin should be regeneration after using 3 times. As the flavonoids content of extraction increase, its antioxidant activity increase. In summary, the X-5 macroporous resin was suitable to purify the total flavonoids of loquat for higher purification fold and higher recovery. Purified extract had higher content of flavonoid and better antioxidant activity, it suggested the flavonoids played an important role in antioxidant activity.
\end{abstract}

\section{Introduction}

Loquat (Eriobotrya japonica (Thunb1.) Lindl.), a kind of subtropical fruit tree in China, has nutritious and delicious fruits with high medical value. Its leaves, fruits, seeds and flowers can be used as medicine, and are usually used to prepare loquat cream and loquat dew [1]. In addition, loquat leave is a traditional Chinese herb with cough-suppressing and phlegm-transforming functions [2-3]. In Japan, loquat leaves are often prepared as loquat tea [4]. The triterpenoids and flavonoids extracted from leaves, flowers and fruits have anti-inflammatory, antioxidant, anticancer, and hypoglycemic effects [5-7] The purification and activity of triterpenoids have been extensively explored. Similarly, its flavonoids and polyphenols also have excellent medicinal efficacy [8-13]. Macroporous resin adsorption method with multiple advantages of removing impurities, high adsorption capacity and repeated utilization during the purification process of natural products has been gradually recognized

\footnotetext{
${ }^{a}$ Corresponding author:kk9096@126.com

This study was supported by Fujian Provincial Key Science and Technology Project (Grant No. 2012N0019), Fujian Provincial Natural Science Foundation (Grant No. 2012D100), Fujian Provincial Department of Education Project (Grant No. JB12204) and The Undergraduate Scientific and Technology Innovation Project of Fujian Province, China (201511312058).
} 
and applied[14-17]. Many experiments show that macroporous resin has excellent separation efficiency for flavonoids [18-20]. The purification and enrichment of flavonoids through macroporous resin can provide a basis for the further exploitation and utilization of loquat leaves.

In the present study, ethanol extraction method was used for obtaining the crude extract of total flavonoids from loquat leaves. The purification efficiency of 6 kinds of macroporous resins for the crude extract of total flavonoids was compared to realize the screening of optimal resin and purification process. The antioxidant activities of purified total flavonoids were evaluated through DPPH and FRAP analysis, which provided a reference for industrial production of total flavonoids from loquat leaves.

\section{Material and methods}

\subsection{Materials and reagents}

Fresh and mature loquat leaves collected from Putian, Fujian, were subjected to wiping and natural drying as well as pulverizing to obtain the dried powder.

The specific parameters of macroporous resins including ADS-7, ADS-17, AB-8, D-101 and X-5 (Nankai University), as well as DM-130 (Shanxing Resin Co., Ltd.) were shown in Table 1. Ursolic acid, 1,1-diphenyl-2-picryl hydrazine (DPPH), terpyridine triazine (TPTZ), Folin-ciocalteu reagent and gallic acid were purchased from Sigma Company. Rutin was purchased from Sinopharm Chemical Reagent Co., Ltd.. Other reagents were of analytical grade.

Table 1. The table of specific parameters of 6 kinds macroporous resins.

\begin{tabular}{cccccc}
\hline $\begin{array}{c}\text { Resin } \\
\text { type }\end{array}$ & Polar & Colour & $\begin{array}{c}\text { Particle } \\
\text { diameter }(\mathrm{mm})\end{array}$ & $\begin{array}{c}\text { Specific surface } \\
\text { area }\left(\mathrm{m}^{2} / \mathrm{g}\right)\end{array}$ & $\begin{array}{c}\text { Average pore } \\
\text { diameter(nm) }\end{array}$ \\
\hline ADS-7 & Polar & Pale yellow & $0.3-1.25$ & $100-120$ & $25-30$ \\
ADS-17 & Medium polarity & Milky white & $0.3-1.25$ & $90-150$ & $25-30$ \\
DM-130 & Weak polarity & Milky white & $0.3-1.25$ & $500-550$ & $90-100$ \\
AB-8 & Weak polarity & Milky white & $0.3-1.25$ & $480-520$ & $13-14$ \\
D101 & Non polarity & Milky white & $0.3-1.25$ & $600-700$ & $10-12$ \\
X-5 & Non polarity & Milky white & $0.3-1.25$ & $500-600$ & $29-30$ \\
\hline
\end{tabular}

\subsection{Methods}

\subsubsection{Preparation of loquat leaf extract}

Approximately $1 \mathrm{~kg}$ of loquat leaf dried powder was subjected to the extraction by $95 \%$ industrial alcohol with the material-liquid ratio of 1:10 for three repeats. The extract was condensed until no taste of alcohol under the reduced pressure condition. The appropriate amount of distilled water was added to the crude extract for standing and the formation of a large amount of precipitate. The mixture was subjected to filtration to obtain the supernatant and precipitate. The supernatant was condensed to a certain concentration as the sample solution for the purification of flavonoids, and the sample solution was stored at $4{ }^{\circ} \mathrm{C}$ for use.

\subsubsection{Establishment of standard curve and determination of total flavonoids in samples}

The standard curve of total flavonoid content was established according to the previous methods from Hong et al [8]. Rutin was used as the standard sample. The sodium nitrite-aluminum nitrate method was applied. The stock standard solution of rutin at the appropriate amount was diluted by $30 \%$ ethanol to $1 \mathrm{~mL}$. Then, $60 \mu \mathrm{L}$ of $5 \%$ sodium nitrite was added to the diluted rutin standard solution with the standing for $5 \mathrm{~min}$. Similarly, $60 \mu \mathrm{L}$ of $10 \%$ aluminum nitrate was added with the standing 
for $6 \mathrm{~min}$, and $400 \mu \mathrm{L}$ of $1.0 \mathrm{~mol} / \mathrm{L}$ sodium hydroxide and $480 \mu \mathrm{L}$ of $30 \%$ ethanol were sequentially added with the standing for 15-20 min. Finally, the optical density (OD) values of rutin standard solutions with various concentrations at $510 \mathrm{~nm}$ were determined and the standard curve was established according to the OD values from gradient concentrations. The tested samples were diluted to a certain concentration, and their absorbance was measured with three repeats in the same manner. The contents of total flavonoids in tested samples were calculated according the standard curve of rutin.

\subsubsection{Screening of optimal macroporous resin}

Static adsorption and desorption of macroporous resins: Totally $1 \mathrm{~g}$ of pretreated ADS-7, ADS-17, DM-130, AB-8, D-101 and X-5 resins was mixed with $20 \mathrm{~mL}$ of sample solution, respectively. The adsorption was conducted at $25{ }^{\circ} \mathrm{C}$ water bath for $6 \mathrm{~h}$. Then, the contents of total flavonoids were determined to calculate the absorption amount of flavonoids. The resin was washed by water and subjected to desorption in $95 \%$ ethanol. The contents of flavonoids in desorption solution was determined to calculate the desorption rate. All experiments are in triplicate.

Dynamic adsorption and desorption of resins: Totally $14 \mathrm{~mL}$ of resin suspension was packed into the column $(10 \mathrm{~mm} * 200 \mathrm{~mm})$ through wet-packing method. The sample loading and absorption were conducted with sample solution of $50 \mathrm{~mL}$ and flow rate of $2 \mathrm{BV} / \mathrm{h}$, and column washing with columnwashing water of $3 \mathrm{BV}$ and flow rate of $2 \mathrm{BV} / \mathrm{h}$. The contents of total flavonoids in effluent and distilled water eluent were determined to calculate the adsorption rate. The desorption was conducted using $95 \%$ ethanol for washing column at the flow rate of $1.5 \mathrm{BV} / \mathrm{h}$. The contents of total flavonoids in the recycled $95 \%$ ethanol was determined to calculate the desorption rate.

\subsubsection{Observation of static behavior from selected resin}

A total of $1 \mathrm{~g}$ of selected resin was sequentially subjected to pre-treatment, the addition of sample solution $(20 \mathrm{~mL})$ at various concentrations. The absorption was conducted in $25^{\circ} \mathrm{C}$ water bath for $6 \mathrm{~h}$. Then, the contents of total flavonoids were determined to calculate the absorption amount and establish an isotherm curve of static absorption at $25^{\circ} \mathrm{C}$.

Similarly, a total of $1 \mathrm{~g}$ of selected resin was sequentially subjected to pre-treatment, the addition of sample solution $(20 \mathrm{~mL})$. The absorption was conducted in $25{ }^{\circ} \mathrm{C}$ water bath, and the sample collection was conducted with the time interval of $1 \mathrm{~h}$. Then, the contents of total flavonoids were determined to calculate the absorption amount and establish an absorption dynamic curve.

Moreover, $1 \mathrm{~g}$ of resin with the adsorption of flavonoids was subjected to the gradient desorption through $20 \mathrm{~mL}$ of ethanol at various concentrations. The contents of total flavonoids in desorption solution were determined to calculate the desorption rate.

\subsubsection{Observation of dynamic behavior from selected resin}

Totally $20 \mathrm{~mL}$ of resin suspension was packed into the column $(10 \mathrm{~mm} * 300 \mathrm{~mm})$ through wetpacking method. The sample solution was loaded to the column at the flow rate of $0.5 \mathrm{BV} / \mathrm{h}$. The effluent was collected at each fraction of $10 \mathrm{~mL}$ and the contents of total flavonoids in effluent solutions were determined to draw a leakage curve.

Totally $20 \mathrm{~mL}$ of resin suspension was packed into the column $(10 \mathrm{~mm} * 300 \mathrm{~mm})$ through wetpacking method. The sample solution was loaded to the column at the flow rate of $0.5 \mathrm{BV} / \mathrm{h}$ until the termination of leakage. Then, the elution was conducted by $5-70 \%$ ethanol in a gradient elution manner. The contents of total flavonoids in eluent solutions were determined to draw an elution curve.

\subsubsection{Determination of purification efficiency and stability of selected resin}


Totally $20 \mathrm{~mL}$ of resin suspension was packed into the column $(10 \mathrm{~mm} * 300 \mathrm{~mm})$ through wetpacking method. According to the optimal sample loading, impurity cleaning and elution process, the eluent was collected, concentrated and dried to obtain a purified product. The appropriate amount of the purified product was dissolved in methanol to determine the content of total flavonoids and to calculate the purity and purification factor of purified total flavonoids.

In addition, the selected resin without regeneration was used to repeat above elution process to determine the recovery rate of total flavonoids, which can be used for evaluating the number of repeated use.

\subsubsection{Antioxidant activity of total flavonoids from loquat leaves}

DPPH method: According to the method from Benzuie and Strain [21], the sample solutions before and after purification by macroporous resin were diluted as 5 concentration gradients. Then, $0.2 \mathrm{~mL}$ of each diluted sample was mixed with $1.98 \mathrm{~mL}$ of DPPH ethanol solution for 30 min reaction. The OD values of the tested samples with various concentrations after reaction were determined to calculate $\mathrm{DPPH}$ radical scavenging rate, as expressed in $\mathrm{EC}_{50}(\mathrm{mg}$ powder $/ \mathrm{mL}$ extract, scavenging rate at the extract concentration of 50\%).

FRAP method: According to the method from Benzuie and Strain [21], the purified flavonoids solution was subjected to the $50-80$ fold dilution. Then, $0.2 \mathrm{~mL}$ of diluted flavonoids solution was mixed with $1.8 \mathrm{~mL}$ of TPTZ working solution for the reaction at $37{ }^{\circ} \mathrm{C}$ for $10 \mathrm{~min}$. The OD value of the mixture after reaction at $593 \mathrm{~nm}$ was determined to calculate the total reducing power, as expressed in the equivalent $\mathrm{FeSO}_{4} \mu \mathrm{moL} / \mathrm{g}$ dried power.

\section{Results and discussion}

\subsection{Standard curve of total flavonoids}

The sodium nitrite-aluminum nitrate method was applied to establish the standard curve using rutin as the standard sample, the absorbance value from rutin at various concentrations as the Y-axis, and absolute content of rutin $(\mathrm{mg})$ as the $\mathrm{X}$-axis. The linear regression equation was determined as $\mathrm{y}=$ $5.82 \mathrm{x}-0.0056(\mathrm{R}=0.9993)$.

\subsection{Screening for resin type}

\subsubsection{Static adsorption and desorption of resins}

When the initial concentration of total flavonoids from loquat leaves was $8.33 \mathrm{mg} / \mathrm{mL}$, the results for dynamic absorption and desorption of 6 kinds of resins were shown in Table 2. Among these resins, resin ADS-7 revealed the highest absorption rate, which reached up to 79.5\%; however, the desorption rate of resin ADS-7 was only 10.09\%, and it is difficult to complete the recovery of flavonoids. Therefore, resin ADS-7 is not suitable for the purification of flavonoids. Based on the analysis of static absorption data, the absorption rates of resins X-5 and DM-130 were just a little lower than that of resin ADS-7, but resin X-5 revealed the significantly higher desorption rate than resin DM-130. Taking the comprehensive consideration, resin X-5 should be the best one.

\subsubsection{Dynamic adsorption and desorption of resins}

The dynamic and static absorption and desorption of 6 kinds of resins exhibited the similar results, and the absorption and desorption rates were shown in Table 2. The highest absorption rate and the lowest desorption rate of resin ADS-7 indicated its large dead absorption and low recovery for flavonoids so that it is not suitable for the purification of flavonoids from loquat leaves. On the other 
hand, resin X-5 revealed a higher adsorption rate and desorption rate. Based on the comprehensive analysis of dynamic and static absorption and desorption efficiency, resin X-5 among these 6 kinds of resins is the best resin for the purification of flavonoids from loquat leaves.

Table 2. The results of static/dynamic absorption and desorption of 6 kinds of resins.

\begin{tabular}{ccccc}
\hline Resin type & $\begin{array}{c}\text { Static } \\
\text { adsorption rate (\%) }\end{array}$ & $\begin{array}{c}\text { Static } \\
\text { resolution ratio (\%) }\end{array}$ & $\begin{array}{c}\text { Dynamic } \\
\text { adsorption rate (\%) }\end{array}$ & $\begin{array}{c}\text { Dynamic } \\
\text { resolution ratio (\%) }\end{array}$ \\
\hline ADS-7 & $79.53 \pm 0.74^{\mathrm{a}}$ & $10.09 \pm 0.21^{\mathrm{d}}$ & 68.54 & 29.65 \\
ADS-17 & $24.73 \pm 0.95^{\mathrm{d}}$ & $63.05 \pm 2.93^{\mathrm{c}}$ & 50.07 & 93.98 \\
DM-130 & $35.85 \pm 0.43^{\mathrm{b}}$ & $70.46 \pm 2.62^{\mathrm{b}}$ & 45.25 & 109.81 \\
AB-8 & $26.96 \pm 0.58^{\mathrm{c}}$ & $68.09 \pm 0.75^{\mathrm{b}}$ & 56.05 & 105.61 \\
D101 & $27.27 \pm 0.29^{\mathrm{c}}$ & $70.72 \pm 1.74^{\mathrm{b}}$ & 60.63 & 105.24 \\
X-5 & $36.90 \pm 0.47^{\mathrm{b}}$ & $82.48 \pm 00.81^{\mathrm{a}}$ & 66.37 & 104.21 \\
\hline
\end{tabular}

Note: The value in the table was were presented as mean \pm standard error, and the different superscript letters indicate that there had a significant different in the same column $(\mathrm{p}<0.05)$.

\subsection{Performance determination of resin X-5}

\subsubsection{Static behavior of resin X-5}
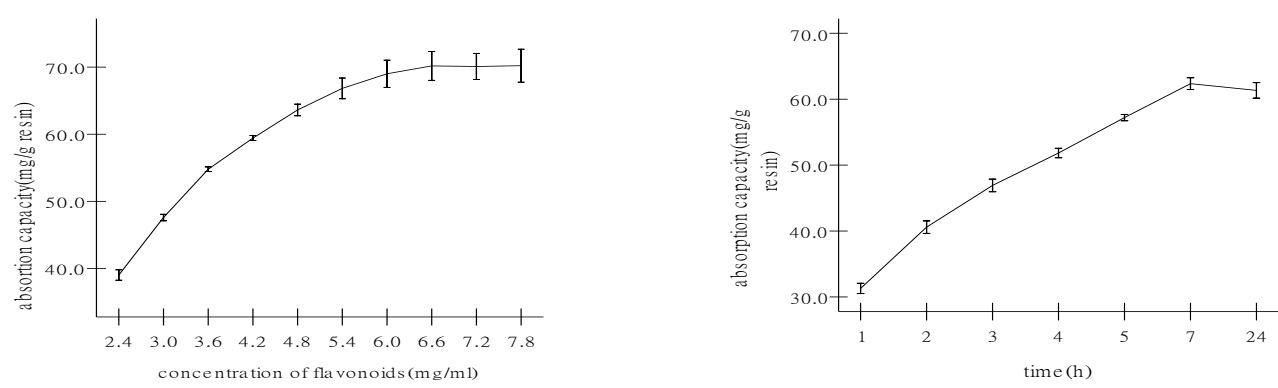

Figure 1. Adsorption isotherm of X-5 resin.

Figure 2. Dynamic curve of X-5 static adsorption.

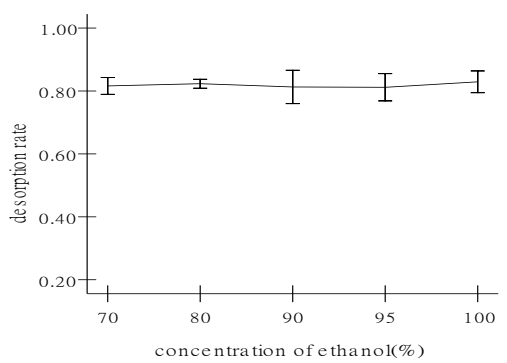

Figure 3. Static desorption curve of $\mathrm{X}-5$ resin.

The static absorption performance of resin X-5 at $25{ }^{\circ} \mathrm{C}$ was determined. As shown in Figure 1-3, the concentration of total flavonoids was $5.4 \mathrm{mg} / \mathrm{mL}$, static adsorption of resin X-5 reached up to equilibrium; meanwhile, the adsorption reached up to the saturation status at the absorption time of 6$7 \mathrm{~h}$. In contrast, the full desorption of flavonoids could be achieved at the ethanol concentration of $70 \%$. 


\subsubsection{Dynamic behavior of resin $X-5$}

The flavonoids solution at the concentration of $5.45 \mathrm{mg} / \mathrm{mL}$ was loaded on resin column. The effluents were collected at the each fraction of $10 \mathrm{~mL}$. The dynamic absorption performance of resin $\mathrm{X}-5$ was determined, as shown in Figure 4. The leakage point was reached when sample solution was loaded at the volume of $30 \mathrm{~mL}$, namely $1.5 \mathrm{BV}$. In addition, the saturation was reached when sample solution was loaded at the volume of $300 \mathrm{~mL}$, namely $15 \mathrm{BV}$. Figure 5 demonstrated the gradient elution curve of ethanol. An elution peak was observed at ethanol concentration of $35-40 \%$, and a complete elution was achieved at ethanol concentration of $65 \%$. In contrast, no elution of flavonoids was observed if ethanol concentration was lower than $15 \%$. Therefore, $15 \%$ ethanol was chosen for the clearance of impurities and $65 \%$ ethanol was chosen for the elution of flavonoids.
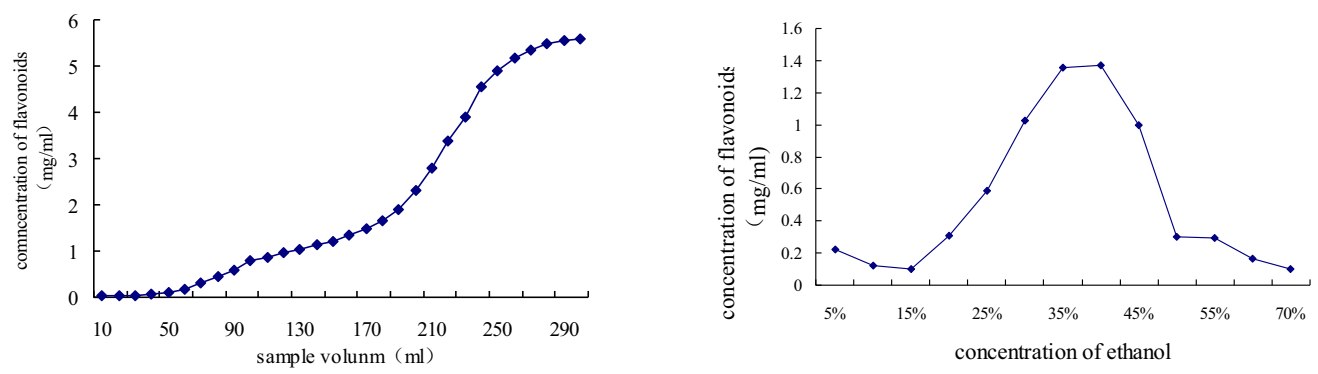

Figure 4. Leakage curve of X-5 dynamic adsorption Figure 5. Gradient elution curve of X-5 dynamic adsorption.

\subsection{Determination of purification efficiency and column efficiency for optimal process}

Totally $20 \mathrm{~mL}$ of X-5 resin suspension was pocked into the column through wet-packing method. The sample solution with the initial flavonoids concentration of $5.47 \mathrm{mg} / \mathrm{mL}$ was loaded, and the loaded volume was $1.5 \mathrm{BV}(30 \mathrm{~mL})$. After the loading of sample solution was completed, $15 \%$ ethanol was used for the clearance of impurities and $65 \%$ ethanol was used for the elution of flavonoids. The purified product was obtained through elution, condensation and drying, which was used for the determination of purification efficiency. Results indicated that the recovery rate of total flavonoids was $81.08 \%$, the purity of total flavonoids was $82.20 \%$ and the purification factor was up to 2.14 .

Table 3. Effect of repetition loading on the stability of the column.

\begin{tabular}{ccccccc}
\hline Loading times & 1 & 2 & 3 & 4 & 5 & 6 \\
\hline Adsorption rate $(\%)$ & 95.28 & 95.09 & 93.94 & 90.70 & 87.86 & 84.36 \\
Loss of flavonoids(\%) & 9.26 & 9.82 & 10.23 & 11.43 & 12.69 & 13.99 \\
Recovery rate of flavonoids(\%) & 79.96 & 73.25 & 68.41 & 62.07 & 55.73 & 46.78 \\
\hline
\end{tabular}

Continuous sample loading, impurity cleaning and elution were conducted to evaluate the column efficiency. As shown in Table 3, the repeated use of resin column could result in a gradual decrease in absorption capacity and recovery rate of flavonoids. If the number of continuous sample loading was 3 , the recovery rate of flavonoids revealed the reduction by $10 \%$. Therefore, the repeat use of resin column was more than 3 times, the clearance of impurities and the regeneration of resin column are required to improve the absorption capacity of resin to total flavonoids.

\subsection{Antioxidant activity of total flavonoids from loquat leaves}

The ethanol solution of DPPH as the standard sample was diluted to a series of concentrations. The OD values of diluted DPPH standard solutions were determined at $517 \mathrm{~nm}$. The DPPH standard equation was obtained to be $\mathrm{y}=30.951 \mathrm{x}+0.0030\left(\mathrm{R}^{2}=0.9993\right)$ using DPPH concentration $(\mu \mathrm{g} / \mathrm{mL})$ 
as the $\mathrm{X}$-axis and absorbance value as the $\mathrm{Y}$-axis. In addition, $0.2 \mathrm{~mL}$ of $\mathrm{FeSO}_{4}$ solution with various concentrations was added to $1.8 \mathrm{~mL}$ of TPTZ working solution for determining the absorbance at 593 $\mathrm{nm}$. The FRAP standard curve was obtained to be $\mathrm{y}=16.212 \mathrm{x}-0.0608\left(\mathrm{R}^{2}=0.9940\right)$ using ferrous sulfate content ( $\mu \mathrm{mol})$ as the $\mathrm{X}$-axis and absorbance as the $\mathrm{Y}$-axis.

The contents and antioxidant performance of crude flavonoids and purified flavonoids were determined, as shown in Table 4. Subjected to the purification by macroporous resin, the contents of purified flavonoids exhibited a significant increase. Meanwhile, the FRAP value also revealed a significant increase, suggesting the significant enhancement of total reducing power. In addition, the $\mathrm{EC}_{50}$ of DPPH clearance revealed a significant decrease, suggesting the enhanced scavenging capacity to free radicals. All of these results indicated that the content of total flavonoids in loquat leaves was consistent with its antioxidant capacity; thereby flavonoids play important roles in anti-oxidation.

Table 4. The contents and antioxidant performance of crude flavonoids and purified flavonoids.

\begin{tabular}{cccc}
\hline Items & $\begin{array}{c}\text { Flavonoid content } \\
(\mathrm{g} / \mathrm{g} \mathrm{DW})\end{array}$ & $\begin{array}{c}\text { FRAP } \\
\left(\mathrm{FeSO}_{4} \mathrm{mmol} / \mathrm{g}\right)\end{array}$ & $\begin{array}{c}\mathrm{DPPH} \\
\left(\mathrm{EC}_{50} \mathrm{mg} / \mathrm{g}\right)\end{array}$ \\
\hline purified flavonoids & $0.839 \pm 0.012^{\mathrm{aA}}$ & $4.559 \pm 0.009^{\mathrm{aA}}$ & $0.360 \pm 0.02^{\mathrm{a}}$ \\
\hline Crude flavonoids & $0.385 \pm 0.005^{\mathrm{bB}}$ & $2.771 \pm 0.002^{\mathrm{bB}}$ & $0.782 \pm 0.12^{\mathrm{b}}$ \\
\hline
\end{tabular}

Note: The same column of different lowercase letters indicate significant difference $(\mathrm{p}<0.05)$, and capital letters indicate extremely significant difference $(\mathrm{p}<0.01)$.

\section{Discussions}

In the present study, the preparation of crude extract from loquat leaves was subjected to cold extraction through 95\% industrial ethanol with three repeats, condensation under reduced pressure, and precipitation through sitting down. The precipitate and filtrate has high contents of total terpenes and flavonoids. The yields of flavonoids and total terpenes in precipitate are $1.22 \%$ and $3.57 \%$, respectively; the yields of flavonoids and total terpenes in extract are $3.28 \%$ and $1.90 \%$, respectively. In addition, the yields of flavonoids and total terpenes in filtrate before condensation are $4.05 \%$ and $1.90 \%$, respectively. However, the content of flavonoids in extract after drying was decreased, and the content of total terpenes was no change, suggesting that flavonoids may be unstable due to oxidation. Therefore, the water-soluble filtrate after extraction and condensation should be the major sample solution for purifying total flavonoids from loquat leaves.

The flavonoids from loquat leaves not only have antioxidant activity, but also have antiinflammatory activity [22]. In this study, the extract from loquat leaves has the enrichment of flavonoids after cold ethanol extraction. The purification process of the extract with enriched flavonoids through macroporous resin was optimized. Based on the experiments of static and dynamic absorption and desorption, resin X-5 was the optimal one; meanwhile, the optimal extraction process for total flavonoids from loquat leaves has been established through analyzing the static and dynamic absorption and desorption behaviors of resin X-5. Experimental results indicated that resin X-5 has better absorption and desorption capacity for total flavonoids from loquat leaves when compared with other 5 kinds of macropourous resins. When the concentration of total flavonoids reached up to 5.4 $\mathrm{mg} / \mathrm{mL}$ at $25{ }^{\circ} \mathrm{C}$, the maximum absorption amount of resin X-5 for total flavonoids was up to 62.32 $\mathrm{mg} / \mathrm{g}$. Subjected to the impurity clearance by $15 \%$ ethanol and elution by $65 \%$ ethanol during the optimal purification process of macroporous resin, the purity of total flavonoids from loquat leaves can reach up to $82.20 \%$ with the purification factor of 2.14 , which reveals the higher purity than other reported purification methods[23-24]. Moreover, the purification through macroporous resin could result in the obvious enrichment of flavonoids, thereby greatly improving the antioxidant capacity, and revealing the important roles of flavonoids in anti-oxidation. 


\section{References}

1. S.M. Lu, W.H.Wang, M.Y. Zheng, Chin. J. Biol. Progr. 6, 453-458 (2013).

2. S.Q. Lin, Acta. Hortic. 750, 37-44 (2007).

3. J.Y. Lin, C.Y.Tang, Food Chem. 107, 1587-1596(2008).

4. H. Tan, S.Furuta, T. Nagata, K.Ohnuki, T.Akasaka, B.Shirouchi, M.Sato, R. Kondo, K. Shimizu, J. Agric. Food Chem. 4, 836-41 (2014).

5. D.Dufour, A.Pichette, V. Mshvildadze, M.E. Bradette-Hébert, S.Lavoiem, A. Longtin, C. Laprise, J.Legault, J. Ethnopharmacol. 111, 22-28 (2007).

6. J.B.Wu, Y.H.Kuo, C.H.Lin, H.Y.Ho, C.C. Shih, J. Agric. Food Chem. 62, 10717-10726 (2014).

7. J.H.Ju, L. Zhou, G. Lin, D. Liu, L.W. Wang, J.S. Yang, Chin. J. Chin. Pharm. J. 38, 752-757 (2003).

8. Y. P.Hong, S. Q. Lin, Y. M. Jiang, M. Ashraf, Plant. Food Hum. Nutr. 63, 200-204(2008).

9. Y. Huang, J. Li, Q. Cao, S.C. Yu, X.W. Lv, Y.Jin, L. Zhang, Y.H. Zou, J.F. Ge, Life Sci. 78, 2749-2757 (2006).

10. H.A.Jung, J.C.Park, H.Y.Chung, J.Kim, J.S. Choi, Arch. Pharm. Res. 22, 213-218(1999).

11. G.I.Nonaka, I.Nishioka, Chem. Pharm. Bull. 30, 4268-4276 (1982).

12. H.J. You, C.Y. Choi, J. Y. Kim, S.J. Parkk, K.S. Hahm, H.G. Jeong, FEBS Lett. 509, 156-160 (2001).

13. Y.P. Hong, S.H. Huang, H.Y. Cao, S.Q. Lin, Z.L. Liu, Chin. J. Acta Horticulturae Sinica. 36, 898 -904 ( 2009).

14. D.H. Li, Y.Wang, Y.S. Lv, J.H.Liu, L. Yang, S.K.Zhang, Y.Z. Zhuo, Biomed. Res. Int. 2015, 1-9 (2015).

15. R.Yang, D. Meng, Y. Song, J. Li, Y. Zhang, X. Hu, Y. Ni, Q.Li, J. Agric. Food Chem. 60, 84508456 (2012).

16. Z. Yu, L. Liu, Y. Xu, L. Wang, X. Teng, X. Li, J. Dai, Carbohydr Polym. 132, 180-186 (2015).

17. M. Zhuang, M. Zhao, L. Lin, Y. Dong, H. Chen, M. Feng, W. D. Sun, G. Su, Food Chem. 190, 338-44 (2016).

18. Y. Dong, M. Zhao, W. D. Sun, M. Zhuang, H. Chen, M. Feng, L. Lin, Food Chem. 168, 538-545 (2015).

19. J. Kim, M. Yoon, H. Yang, J. Jo, D. Han, Y.J. Jeon, S. Cho, Food Chem. 162, 135-142 (2014).

20. P. Zhao, C. G. Qi, Wang, X. Dai, X. Hou, J. Chromatogr B Analyt Technol. Biomed. Life Sci. 1007, 8-17 (2015).

21. I.F. Benzuie, J.J. Strain, Anal. Biochem. 239, $70-76$ (1996).

22. K. Maher, B.A. Yassine, B. Sofiane, Afr. Health. Sci. 15, 613-20 (2015).

23. Y. Liu, X.T. Zhu, Chin. J. Exp. Traditional Med. Formulae. 18, $55-57$ (2012).

24. H. Lv, J.H.Teng, J. Chen, L. Ma, B.R. Ren, W.L. Li, Chin. J. Mod. Appl. Pharm. 31, 40-44 (2014). 